
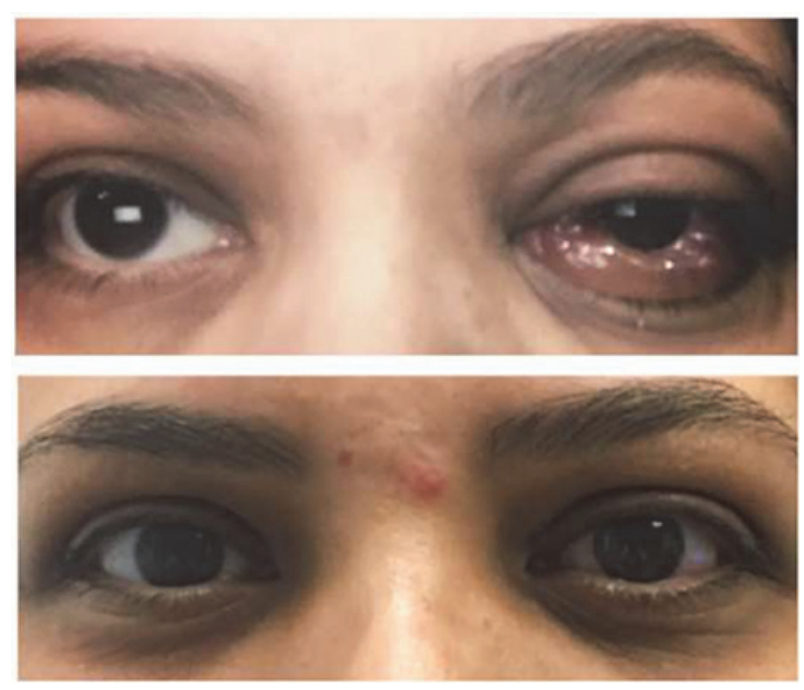

Abstract E-025 Figure 1 A 45-year-old female presented with one month of left eye swelling, injection, and pain with associated double vision (top image). Two months after the transorbital CCF embolization, she had resolution of all ophthalmologic symptoms (bottom image)

elevated intraocular pressure from complete occlusion of the inferior orbital vein, though no visual sequelae were noted at follow up and intraocular pressure normalized over the following weeks. One patient who underwent multiple interventions - and ultimately fistula obliteration-progressed to blindness in the affected eye as her treatment course was prolonged.

Conclusion Direct percutaneous transorbital puncture is a generally safe and effective means of accessing and treating CCFs. Ophthalmologic cut-down is unnecessary in most cases, and inaccessibility of the superior or inferior orbital veins can be overcome through direct transorbital puncture of the cavernous sinus.

Disclosures B. King: None. J. Steinberg: None. A. Wali: None. R. Rennert: None. D. Santiago-Dieppa: None. J. Pannell: None. A. Khalessi: None. S. Olson: None.

\section{E-026 AROUND THE BLIND CORNER OF THE MOUTH: A GLIDE- SCOPE DIRECTED ACCESS TO OROPHARYNGEAL VENOLYMPHATIC MALFORMATIONS}

${ }^{1} \mathrm{~T}$ Le*, ${ }^{1} \mathrm{~T}$ Akinpelu, ${ }^{1} \mathrm{D}$ Hallam, ${ }^{2} \mathrm{~S}$ Vaidya, ${ }^{1} \mathrm{~B}$ Ghodke. ${ }^{1}$ Interventional Neuroradiology, University of Washington, Seattle, WA; ${ }^{2}$ Interventional Radiology, University of Washington, Seattle, WA

\subsection{6/neurintsurg-2020-SNIS.62}

Introduction/Purpose Low flow vascular malformations can present anywhere in the body with high prevalence in the head and neck region. Locations in the oropharynx at the base of the tongue or peritonsillar space can complicate percutaneous sclerosant application for minimally invasive treatment of symptomatic lesions. Both anterior transfacial and posterior transcondylar approaches can be limited by a small working window of access to the lesion with potential high risk complication to adjacent surrounding sensitive organs and major vasculatures. Thus, limited visibility for direct percutaneous access into low flow vascular malformation in the oropharynx may exclude a large population of patients to an alternative option to treatment other than surgery. Thus, the purpose of our study is to describe a novel approach using the GlideScope, a commonly use device during difficult airway intubation, for access into otherwise difficult and often out of reach low flow vascular malformation in the oropharyngeal space.

Materials/Methods Single center retrospective review of 4 cases of oropharyngeal venolympathic malformation, 3 of which utilized a combined Glide-Scope directed and fluoroscopic guided sclerotherapy technique while the remaining were performed via the transfacial or transcondylar approach.

Results Please see table 1.

\begin{tabular}{lccc}
\multicolumn{3}{l}{ Abstract E-026 Table 1} \\
\hline Patient & $\begin{array}{c}\text { Fluoro Time } \\
\text { (minutes) }\end{array}$ & $\begin{array}{c}\text { Procedure Time } \\
\text { (hours) }\end{array}$ & Patient Response \\
\hline 1 & 1.30 & 2.3 & Asymptomatic. \\
2 & 3.49 & 1.1 & $\begin{array}{c}\text { Decreased lesion size. } \\
\text { Asymptomatic. }\end{array}$ \\
3 & 1.87 & 0.5 & Asymptomatic. \\
\hline
\end{tabular}

Conclusion Symptomatic low flow oropharyngeal vascular malformations can can be technically challenge during initial access for percutaneous sclerotherapy. Our single center retrospective experience reviewed 4 cases, 3 of which were via a novel approach in gaining direct access into oropharyngeal venolymphatic malformations utilizing a video assisted GlideScope. We demonstrate high technical success, reduced fluoroscopic and procedural time, and excellent patient response.

Disclosures T. Le: None. T. Akinpelu: None. D. Hallam: None. S. Vaidya: None. B. Ghodke: None.

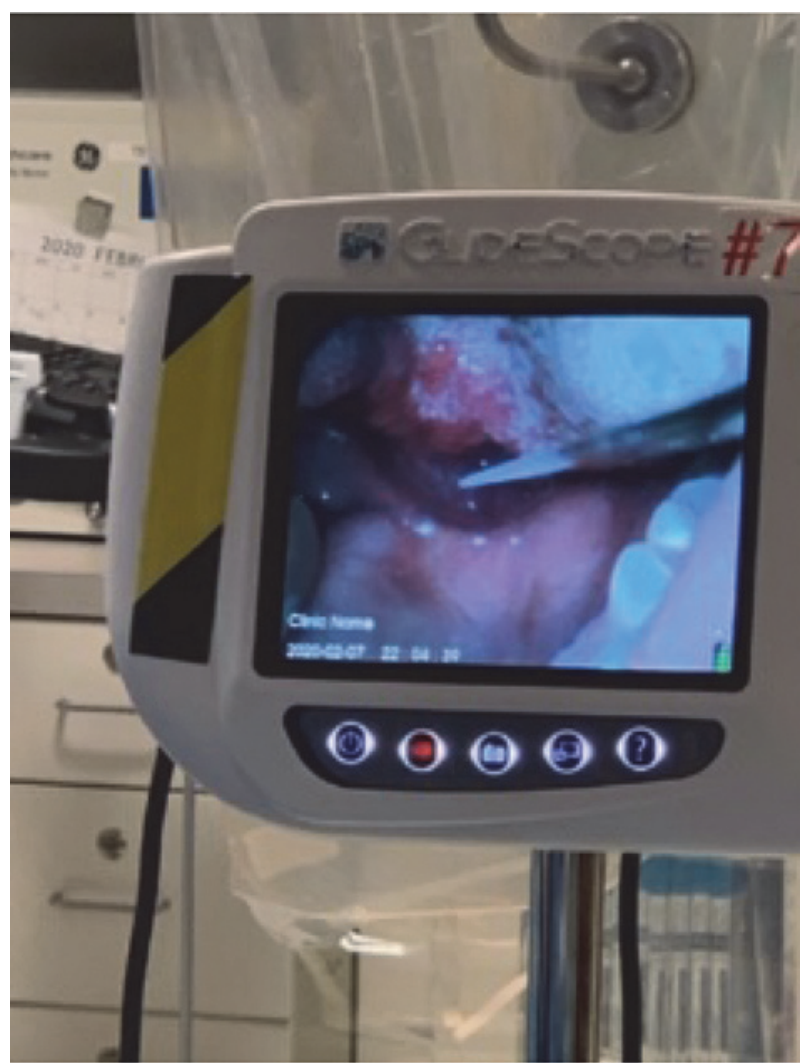

Abstract E-026 Figure 1 